\title{
A Case Report of Prototheca Meningitis in China
}

\author{
Li YT, Zeng YF, Ning G, Gan WQ and Lin CS ${ }^{*}$
}

Department of Infectious Diseases, The Third Affiliated Hospital of Sun Yat-Sen University, Guangzhou 510630, China

*Corresponding author: Lin CS, Department of Infectious Disease, The Third Affiliated Hospital of Sun Yat-Sen University, Guangzhou 510630, China, Tel: 020-85253008; Fax: 020-85252559; E-mail: linchaoshuang@126.com

Received date: March 21, 2016; Accepted date: May 02, 2016; Published date: May 09, 2016

Copyright: $\odot 2016 \mathrm{Li} \mathrm{YT}$, et al. This is an open-access article distributed under the terms of the Creative Commons Attribution License, which permits unrestricted use, distribution, and reproduction in any medium, provided the original author and source are credited.

\begin{abstract}
Human prototheca meningitis was rarely reported in the world; here we present another case report. A 23 year old young man was admitted to department of infectious disease of the third affiliated hospital of Sun Yat-sen university, complaining of fever, headache and vomiting. Before admission, he had been diagnosed as tuberculous meningitis. After taking chemical therapy with antituberculous drugs combined with adrenocortical hormones for two months, his condition deteriorated. Cerebrospinal fluid (CSF) was collected through lumbar punture for the patient, and prototheca species, which was sensitive to amphotericin B and nystatin, was discovered in CSF after culture. During hospitalization, the patient's condition once ameliorated when the total amount of amphotericin B reached to $2400 \mathrm{mg}$ three months later. However, the secondary bacterial infection in central nervous system, abdomen, and lungs might contribute to the death of the patient when amphotericin B reached to $6000 \mathrm{mg}$ after 11 months. Prototheca species, though rarely been described, may also have a significant pathogenic potential in human.
\end{abstract}

Keywords: Prototheca; Meningitis; Amphotericin B; Nystatin

\section{Introduction}

Prototheca infections were mainly seen in animals, while In humans, prototheca mostly cause cutaneous infections, $25 \%$ cases were olecranon bursitis, and systemic infection including Chronic peritonitis in peritoneal dialysis patients, secondary infection of AIDS or Chronic meningitis, etc., were rare disseminated diseases. Davies et al. [1] reported the first human infection with this specie in 1964 and since then sporadic cases of prototheca infection were described in different parts of the world. And then, the first descript of prototheca meningitis was reported by Kaminski in 1992 [2]. In 1996, the second case of meningitis was described in Japan and the infection persisted for 6 years [3]. In China, the first detailed report of meningitis was caused by $P$. wickerhamii which occurred in 2007 and was successfully treated with AMB and ITZ [4]. And Prototheca wickerhamii as a cause of neuroinfection in a child with congenital hydrocephalus was the first case of human protothecosis in Poland [5]. To our knowledge, we reported the 5th case of human prototheca meningitis in the literature.

\section{Case Report}

A 23 year old young man was admitted to department of infectious disease of the third affiliated hospital of Sun Yat-sen university, complaining of fever, headache and vomiting. Before admission, he had been diagnosed as tuberculous meningitis. After taking chemical therapy with antituberculous drugs combined with adrenocortical hormones for two months, his condition deteriorated. CT scan showed expansion of lateral and the third ventricle, and edema of the brain. The patient was an immunocompetent adult before the infection and declared no abnormality in his past medical history. Physical examination at admission showed this patient had a temperature of $38^{\circ} \mathrm{C}$, respiratory rate: 20 times per min, pulse rate: 86 times per min and with blood pressure 105/70 mm Hg. Besides, neurological examination revealed that $\mathrm{He}$ had a stiff neck with Kernig's sign positive while Babinski's sign was negative. Physical examination of lung, heart, and abdomen was normal.

Laboratory examinations of this patient at admission showed a normal blood routine, but an abnormal cerebrospinal fluid (CSF) with red blood cell $(\mathrm{RBC})$ count: $381 \times 10^{6} / \mathrm{L}$, white blood cell $(\mathrm{WBC})$ count: $317 \times 10^{6} / \mathrm{L}$, sodium chloride: $123.5 \mathrm{mmol} / \mathrm{L}$, glucose: 0.1 $\mathrm{mmol} / \mathrm{L}$, protein: $1.44 \mathrm{~g} / \mathrm{L}$, and neither cryptococcus or acid-fast bacilli was discovered by smear or culture. CT scan showed communicating hydrocephalus.

After being admitted to the hospital, the patient was given Isoniazid (INH), Rifampicin (RFP), Pyrazinamide (PZA) and Ethambutol (EMB) for two weeks. The dosage of Corticoid was reduced gradually. OMMAYA Sack was put in subcutaneous tissue of the brain through lumbar punture to drain cerebrospinal fluid to the sack for latter analysis or test, and prototheca species, which was sensitive to amphotericin B and nystatin, was discovered in CSF after culture.

Consequently, intravenous administration of amphotericin B (from $1 \mathrm{mg}$ per day increased gradually to $30-35 \mathrm{mg}$ per day) was given to the patient, combined with 5-FC (100 mg, three times a day) orally. Three month later (Total dosage of amphotericin B reached to $2400 \mathrm{mg}$ ), the patient's condition ameliorated, which was confirmed by laboratory examinations of CSF with red blood cell count significantly decreased to $26 \times 10^{6} / \mathrm{L}$, and white blood cell counts decreased to $20 \times 10^{6} / \mathrm{L}$; sodium chloride: $130 \mathrm{mmol} / \mathrm{L}$; Glucose: $2.9 \mathrm{mmol} / \mathrm{L}$; protein: $0.39 \mathrm{~g} / \mathrm{L}$; culture of fungi (-). However, nephrotoxicity of amphotericin B occurred and the patient had fever again after four months taking the drug, also, culture of CSF showed Staphylococcus warneri. Purulence could be punctured from the tissue around the OMMAYA SACK, and Staphylococcus epidermidis were identified in the purulence after culture. So administration of amphotericin B and 5-FC was ceased. At the same time penicillin was used to relieve bacterial meningitis. Gradually, the patient's temperature descended to normal level. Five months later, Prototheca species was discovered again in the culture of CSF and protein in CSF was $1.39 \mathrm{~g} / \mathrm{L}$, therefore, amphotericin B with 5 -FC was given back to the patient. 
In order to reduce the exogenous infection, the OMMAYA SACK was removed and ventricle-abdomen shunt draining CSF to the abdomen was conducted. Six months later, culture of CSF still yielded Prototheca species and Staphylococcus epidermidis, and the patient suffered from severe abdomen pain locating at the left part of abdomen where Ultrasonic scan showed "fluid sonolucent area". Under this condition, secondary peritonitis was considered to cause the abdomen pain, so antibiotics was given and the abdomen pain relieved a month later. After nine months, the patient suffered from fever again and chest $\mathrm{x}$-ray showed lung infection, Prototheca species also could be found in CSF and the concentration of protein was $1.08 \mathrm{~g} / \mathrm{L}$. Unfortunately, the condition of the patient still deteriorated under treatment, and he died eleven months later with the total dosage of amphotericin $B$ reached up to $6000 \mathrm{mg}$.

\section{Discussion}

As an infectious agent, prototheca is a unicellular, round and colorless green alga without flagella (Figure 1). Protothecosis is an uncommon fungal infection most often found as an opportunistic infection in immunosuppressive individuals. Most of the patients have the history of trauma or contacting contaminated water, and $50 \%$ of the patients have immunosuppressive factors. In humans, three clinical manifestations of protothecosis can be observed: 1) cutaneous lesions, 2) olecranon bursitis, 3) systemic protothecosis. To our knowledge, the first documented case was reported in a patient with AIDS and coinfect with Cryptococcus neoformans in New Jersey in 1992 [2]. In this case, the initial CSF culture yielded only Cryptococcus neoformans, $P$. wickerhamii was discovered in CSF after four subsequent lumbar punctures. Autopsy was taken when the patient died, and the alga was isolated from leptomeninges in the brain and spinal cord, in addition, histologic sections from numerous locations of the brain further revealed the masses of cryptococci and prototheca. The second documented case, which reported in Japan in 1996, a patient with chronic meningitis caused by Prototheca wickerhamii had persisted for more than 6 years despite treatment with various antifungal agents. For the last year, the patient had no complaints even though no treatment had been given to [3]. In China, the first detailed report of meningitis caused by $P$. wickerhamii occurred in 2007 [4], in this case, a 24 year old man without any underlying immunodeficient condition presented with 9 month history of meningitis and successfully treated with AMB and ITZ. And in 2012, Prototheca wickerhamii, as a cause of neuroinfection in a child with severs congenital hydrocephalus was the first case of human protothecosis in Poland [5]. After the patient was treated with oral ketoconazole, followed by intravenous fluconazole and amphotericin $\mathrm{B}$, the infection controlled completely. This report focused on the diagnosis of protothecosis and emphasized that a proper diagnosis of protothecosis relied on isolation of the alga in after culture, or recognizing the alga directly in clinical samples. In our case report, the patient was an immunocompetent adult, and didn't suffer from immunosuppressive conditions and had no history of trauma. But in the course of treatment, he had been diagnosed as tuberculous meningitis, and treated with adrenocortical hormones for two months.
In this condition, he seemed to have low immunobility and had secondary infections (bacterial meningitis, peritonitis and lung infection) during hospitalization. Nonetheless, the exact route of infection of this patient remained unclear.

Although there is no standard regiment for the treatment of protothecisis, amphotericin B is thought to be the first choice for Protothecosis. Notably, the duration and effect has not been identified, and treatment of protothecosis remains controversial. We should do our best to avoid the secondary infections during hospitalization in the immunocompromised personers in the future. As far as we know, cured cases of cutaneous protothecosis have been reported in the literature, but no prototheca meningitis cases. One of the cases died in spite of administrating of Amphotericin B. And the other prototheca case could not be eliminated from CSF although he had no complains. In this case, secondary infection of central nervous system, abdomen, and lungs might contribute to the death of the patient.

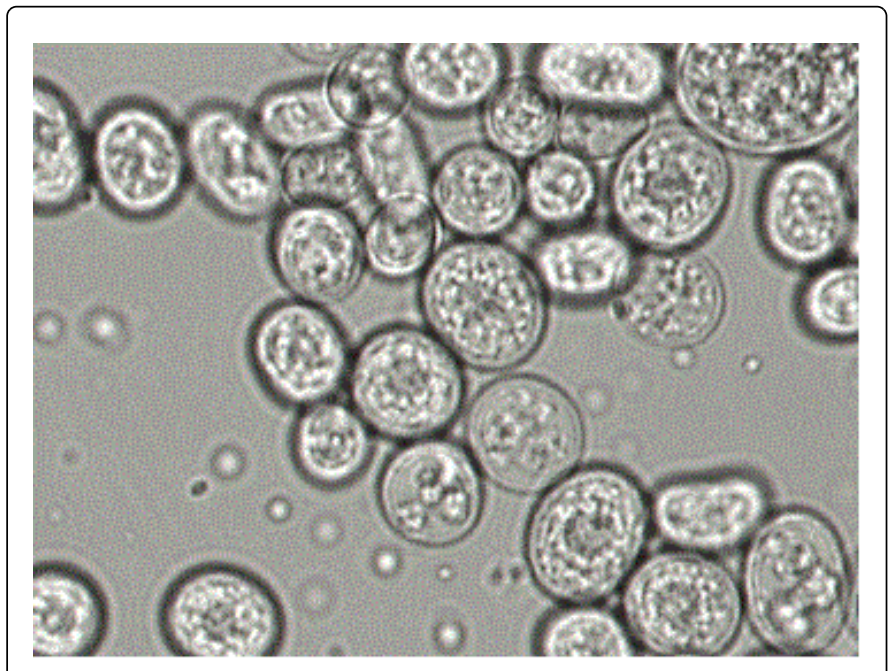

Figure 1: Culture of CSF showed round unicellular of Prototheca.

\section{References}

1. Davies RR, Spencer H, Wakelin PO (1964) A case of human protothecosis. Trans R Soc Trop Med Hyg 58: 448-451.

2. Kaminski ZC, Kapila R, Sharer LR, Kloser P, Kaufman L (1992) Meningitis due to Prototheca wickerhamii in a patient with AIDS. Clin Infect Dis 15: 704-706.

3. Takaki K, Okada K, Umeno M, Tanaka M, Takeda T, et al. (1996) Chronic prototheca meningitis. Scand J Infect Dis 28: 321-323.

4. Zhang QQ, Zhu LP, Weng XH, Li L, Wang JJ (2007) Meningitis due to Prototheca wickerhamii: Rare case in China. Med Mycol 45: 85-88.

5. Zak I, Jagielski T, Kwiatkowski S, Bielecki J(2012) Prototheca wickerhamii as a cause of neuroinfection in a child with congenital hydrocephalus. First case of human protothecosis in Poland. Microbiol Infect 74: 186-189. 